\title{
Unified Model of D-Term Inflation
}

\author{
Valerie Domcke ${ }^{1}$ and Kai Schmitz ${ }^{2}$ \\ ${ }^{1}$ APC / PCCP, Paris Diderot University, 75013 Paris, France \\ ${ }^{2}$ Max-Planck-Institut für Kernphysik (MPIK), 69117 Heidelberg, Germany
}

(Dated: June 29, 2018)

\begin{abstract}
Hybrid inflation, driven by a Fayet-Iliopoulos (FI) D term, is an intriguing inflationary model. In its usual formulation, it however suffers from several shortcomings. These pertain to the origin of the FI mass scale, the stability of scalar fields during inflation, gravitational corrections in supergravity, as well as to the latest constraints from the cosmic microwave background. We demonstrate that these issues can be remedied if D-term inflation is realized in the context of strongly coupled supersymmetric gauge theories. We suppose that the D term is generated in consequence of dynamical supersymmetry breaking. Moreover, we assume canonical kinetic terms in the Jordan frame as well as an approximate shift symmetry along the inflaton direction. This provides us with a unified picture of D-term inflation and high-scale supersymmetry breaking. The D term may be associated with a gauged $U(1)_{B-L}$, so that the end of inflation spontaneously breaks $B-L$ in the visible sector.
\end{abstract}

Cosmic inflation [1] is a successful paradigm in our understanding of the early universe. It is, however, still unclear how to correctly embed inflation into particle physics [2]. One promising ansatz is the idea of hybrid inflation [3], which establishes a connection between inflation and grand unification. Hybrid inflation exits into the subsequent radiation-dominated phase via a waterfall transition. In the context of a given grand unified theory (GUT), this phase transition may then be identified with the spontaneous breakdown of a local GUT symmetry.

Depending on the type of GUT symmetry, the waterfall transition may have important consequences for the particles of the standard model (SM). Here, a prominent example is the spontaneous breaking of $U(1)_{B-L}$ [4], i.e., the Abelian gauge symmetry associated with the difference between baryon number $B$ and lepton number $L$. The end of inflation is then accompanied by the generation of large $L$-violating Majorana masses for a number of right-handed neutrinos, which sets the stage for the type-I seesaw mechanism [5] as well as for baryogenesis via leptogenesis [6]. Hybrid inflation ending in a $B-L$ phase transition, thus, promises to provide an appealing framework for the early universe that not only determines the initial conditions of the hot thermal phase, but which also explains the smallness of the SM neutrino masses.

In its simplest, nonsupersymmetric form, hybrid inflation predicts the primordial scalar power spectrum to be blue-tilted, which is by now observationally ruled out at a level of more than $5 \sigma$ [7]. This problem can be avoided in supersymmetry (SUSY), where scalar and fermion loops generate a logarithmic effective potential. Supersymmetric hybrid inflation comes in two variants, depending on whether the vacuum energy density during inflation is dominated by a nonzero $\mathrm{F}$ term [8] or $\mathrm{D}$ term [9]. In Fterm hybrid inflation (FHI), the inflaton field itself has a large $\mathrm{F}$ term during inflation. In combination with $R$ symmetry breaking, this results in a dangerous supergravity (SUGRA) tadpole term [10], which breaks the rotational invariance in field space, generates a false vac- uum at large field values, and potentially spoils slow-roll inflation. In D-term hybrid inflation (DHI), the superpotential of the inflationary sector has, by contrast, zero vacuum expectation value (VEV) at all times, so that SUGRA corrections tend to become more manageable. Moreover, DHI is based on a nonzero Fayet-Iliopoulos (FI) D term [11] and, hence, does not require a dimensionful input parameter in the superpotential.

In this paper, we shall construct a consistent SUGRA model in which DHI is driven by the D term associated with a local $U(1)_{B-L}$ symmetry. Despite the absence of the inflaton tadpole term, this is still a difficult task for at least five reasons: (i) The consistent embedding of the FI term into SUGRA is a subtle issue that has been the subject of a long debate in the literature. On the one hand, constant, field-independent FI terms always require an exact global symmetry [12], which conflicts with the expectation that quantum gravity actually does not admit such symmetries [13]. On the other hand, field-dependent FI terms (such as those in string theory [14]) imply the existence of a shift-symmetric modulus field [15], which causes cosmological problems [16], as long as it is not properly stabilized [17]. (ii) The sfermions in the minimal supersymmetric standard model (MSSM) carry nonzero $B-L$ charges and, thus, acquire D-term-induced masses during inflation [18]. Some of these masses are tachyonic and may, hence, destabilize the corresponding directions in scalar field space [19]. (iii) General arguments in SUGRA [20] indicate that a nonzero D term is typically accompanied by a comparatively larger $\mathrm{F}$ term, $|F| \gtrsim|D|$. If SUSY breaking is mediated to the visible sector by ordinary gravity mediation [21], this implies that the inflaton picks up a gravity-mediated soft mass of the order of the gravitino mass, $m_{3 / 2} \sim|F| / M_{\mathrm{Pl}}$, that necessarily exceeds the Hubble rate during inflation, $H \sim|D| / M_{\mathrm{Pl}}$. DHI in combination with ordinary gravity mediation, therefore, also faces the $\eta$ slow-roll problem in SUGRA [22]. (iv) In the global-SUSY limit, DHI predicts a scalar spectral index of $n_{s} \simeq 0.98$ [9]. This 
deviates from the latest value reported by the PLANCK collaboration, $n_{s}^{\text {obs }}=0.9677 \pm 0.0060$ [7], by about $2 \sigma$. SUGRA corrections may help to reach better agreement with the data [23]. But in general, realizing a spectral index of $n_{s} \simeq 0.96$ in DHI is a nontrivial task. (v) In its standard formulation, DHI is driven by a $\mathrm{D}$ term associated with a $U(1)$ symmetry that becomes spontaneously broken only during the waterfall transition at the end of inflation. This results in the production of cosmic strings, which impact the scalar power spectrum of the cosmic microwave background (CMB). The recent $\mathrm{CMB}$ bounds on the tension of such cosmic strings [24] severely constrain the parameter space of standard DHI.

We now argue that all of these issues can be remedied as soon as one makes the following three assumptions: (i) The FI term is dynamically generated in the context of dynamical SUSY breaking (DSB) [19]. (ii) DHI is embedded into Jordan-frame SUGRA with canonical kinetic terms for all fields [25]. (iii) There exists an approximate shift symmetry in the direction of the inflaton field [26]. For a more comprehensive account of our idea, see [27].

As far as the generation of the FI term is concerned, we follow the discussion in [19]. We assume that SUSY is broken in a hidden sector by the dynamics of a strongly interacting supersymmetric gauge theory. To this end, we shall employ the Izawa-Yanagida-Intriligator-Thomas (IYIT) model [28] in its $S U(2)$ formulation, i.e., the simplest conceivable DSB model with vector-like matter fields. At high energies, the IYIT model consists of four quark fields $\Psi^{i}$ in the fundamental representation of $S U(2)$. At energies below the dynamical scale $\Lambda_{0}$, these quarks condense into six gauge-invariant meson fields, $M^{i j} \simeq\left\langle\Psi^{i} \Psi^{j}\right\rangle /\left(\eta^{2} \Lambda\right)$, where $\Lambda \simeq \Lambda_{0} / \eta$ and $\eta \simeq 4 \pi$ [29]. The scalar mesons span a quantum moduli space of degenerate supersymmetric vacua, subject to a particular constraint on their Pfaffian, $\operatorname{Pf}\left(M^{i j}\right) \simeq \Lambda^{2}$ [30]. In order to break SUSY in this model, one couples the high-energy theory to a set of six $S U(2)$ singlets, $Z_{i j}$, so as to lift the flat directions in moduli space. At high and low energies, the IYIT superpotential respectively reads as follows,

$$
W_{\mathrm{hid}}^{\mathrm{HE}}=\frac{1}{2} \lambda_{i j} Z_{i j} \Psi^{i} \Psi^{j} \rightarrow W_{\mathrm{hid}}^{\mathrm{LE}} \simeq \frac{1}{2} \lambda_{i j} \Lambda Z_{i j} M^{i j},
$$

where $\lambda_{i j}$ is a matrix of Yukawa couplings. SUSY is now broken à la O'Raifeartaigh [31], as the F-term conditions for the singlet fields $Z_{i j}$ are incompatible with the Pfaffian constraint. A crucial observation for our purposes is that the IYIT model exhibits an axial $U(1)$ flavor symmetry associated with a quark phase rotation, $\Psi^{i} \rightarrow e^{i q_{i} \alpha} \Psi^{i}$. We shall now identify this symmetry with $U(1)_{B-L}$ and promote it to a weakly gauged local symmetry. In doing so, we suppose that two quarks carry charge $q_{0} / 2$, while the other two carry charge $-q_{0} / 2$. In this case, we have to deal with six mesons (and similarly six singlets) with charges $q_{0},-q_{0}$, and four times 0 , respectively. Here, we assign the $B-L$ charges in such a way that the charged mesons, $M_{ \pm}$, have the smallest Yukawa couplings, $\lambda_{ \pm}$. During SUSY breaking, it is therefore the fields $M_{ \pm}$that acquire nonzero VEVs. The neutral mesons and singlets remain stabilized at their origin. In the weakly gauged limit, one finds (see [18, 19, 32, 33] for more details on the dynamics of the IYIT model and its applications),

$$
\left\langle M_{ \pm}\right\rangle=\frac{\lambda}{\lambda_{ \pm}} \Lambda, \quad \lambda=\sqrt{\lambda_{+} \lambda_{-}} .
$$

These VEVs break $B-L$ spontaneously, which results in an effective FI term in the $B-L$ D-term scalar potential,

$$
V_{D}=\frac{g^{2}}{2}\left[q_{0}\left(\left|M_{-}\right|^{2}-\left|M_{+}\right|^{2}\right)+\cdots\right]^{2} .
$$

Here, $g$ denotes the $B-L$ gauge coupling, while the ellipsis stands for all further fields that are charged under $U(1)_{B-L}$. One then obtains for the FI mass scale $\xi$,

$$
\xi=\left\langle M_{-}\right\rangle^{2}-\left\langle M_{+}\right\rangle^{2}=\frac{2}{\rho^{2}}\left(1-\rho^{4}\right)^{1 / 2} \Lambda^{2},
$$

where $\rho=\left[\left(\lambda_{+} / \lambda_{-}+\lambda_{-} / \lambda_{+}\right) / 2\right]^{-1 / 2}$ is a measure for the degeneracy among the Yukawa couplings $\lambda_{+}$and $\lambda_{-}$. For $\lambda_{+} \simeq \lambda_{-}, \rho$ is close to unity; for a strong hierarchy among $\lambda_{+}$and $\lambda_{-}$, it takes a value close to zero. In the following, we will assume that $\lambda_{+}$and $\lambda_{-}$are both of the same order of magnitude. Averaging all possible values of $\rho$ under this assumption (varying $\lambda_{+}$and $\lambda_{-}$on a linear scale) then results in an expectation value of $\langle\rho\rangle \simeq 0.80$.

The FI parameter in Eq. (4) is a field-dependent FI term, as it originates from the VEVs of the two meson fields $M_{ \pm}$. The modulus field associated with this FI term is nothing but the $B-L$ Goldstone multiplet, $A=\left(\left\langle M_{+}\right\rangle M_{+}-\left\langle M_{-}\right\rangle M_{-}\right) / f_{a}$, where $f_{a}$ is the Goldstone decay constant, $f_{a}^{2}=\left\langle M_{+}\right\rangle^{2}+\left\langle M_{-}\right\rangle^{2}$. The pseudoscalar in $A$ is absorbed by the massive $B-L$ vector boson, while the real scalar in $A$ is stabilized by an $\mathrm{F}$ term-induced mass, $m_{F}=\rho \lambda \Lambda$. The same holds true for the fermion in $A$. Owing to the fact that our FI term is generated in conjunction with dynamical SUSY breaking, we therefore do not face any modulus problem. Our model, thus, avoids the problems described in $[12,15]$.

In the SUSY-breaking vacuum at low energies, the IYIT model effectively reduces to the Polonyi model [34] with an effective superpotential of the following form [33],

$$
W_{\text {hid }}=\mu^{2} X+w .
$$

Here, $\mu^{4}=\lambda_{+}^{2}\left\langle M_{+}\right\rangle^{2} \Lambda^{2}+\lambda_{-}^{2}\left\langle M_{-}\right\rangle^{2} \Lambda^{2}=2 \lambda^{2} \Lambda^{4}$ denotes the F-term SUSY-breaking scale, $X=\left(Z_{+}+Z_{-}\right) / \sqrt{2}$ is the Polonyi field, and $w$ is an $R$-symmetry-breaking constant that needs to be added to $W_{\text {hid }}$, so as to achieve zero cosmological constant in the true vacuum.

We now couple the effective Polonyi model in Eq. (5) to SUGRA. In doing so, we shall work in Jordan-frame 
supergravity with canonical kinetic terms [25]. The total Kähler potential of our theory is therefore given as

$$
K_{\mathrm{tot}}=-3 M_{\mathrm{Pl}}^{2} \ln \left(-\frac{\Omega_{\mathrm{tot}}}{3 M_{\mathrm{Pl}}^{2}}\right),
$$

where $\Omega_{\mathrm{tot}}=-3 M_{\mathrm{Pl}}^{2}+F_{\mathrm{tot}}$ is the frame function of the Jordan frame. We assume that the kinetic function $F_{\text {tot }}$ can be split into separate contributions from the hidden, visible, and inflaton sector. Schematically, we may write

$$
F_{\text {tot }}=F_{\text {hid }}+F_{\text {vis }}+F_{\text {inf }}+\frac{1}{M_{*}^{2}} F_{\text {hid }} F_{\text {vis }}
$$

such that the inflaton sector becomes sequestered from the hidden sector [35]. This serves the purpose to protect the inflaton from a SUGRA mass of the order of $m_{3 / 2} \gtrsim$ $H$, which would otherwise spoil slow-roll inflation. Meanwhile, the MSSM sfermions do acquire soft masses via gravity mediation. These may be much larger than $m_{3 / 2}$, provided that the mass scale $M_{*}$ is parametrically smaller than the reduced Planck mass $M_{\mathrm{Pl}} \simeq 2.44 \times 10^{18} \mathrm{GeV}$. In particular, by choosing an appropriate value of $M_{*}$, the MSSM sfermions are also sufficiently stabilized during inflation, even if inflation is driven by a $B-L \mathrm{D}$ term.

For $F_{\text {hid }}=|X|^{2}+$ [other fields], we obtain for the Polonyi VEV during $(f \neq 0)$ and after $(f=0)$ inflation,

$$
\langle X\rangle=\frac{(k-4 / 3)^{1 / 2}}{[(1-f) k-4 / 3] k^{1 / 2}} \frac{2 M_{\mathrm{Pl}}}{\sqrt{3}} .
$$

Here, $f$ is related to the kinetic function of the inflaton field $S, f=\left(F_{\text {inf }}-\partial_{S} F_{\text {inf }} \partial_{S^{\dagger}} F_{\text {inf }}\right) /\left(3 M_{\mathrm{Pl}}^{2}\right) . k$ is a ratio of different contributions to the total Polonyi mass,

$$
k=\left[\left(m_{1 \ell}^{J}\right)^{2}+2 H_{J}^{2}\right] \frac{M_{\mathrm{Pl}}^{2}}{\mu^{4}},
$$

which is typically very large, $k \gg 1$. This reflects the fact that $X$ is stabilized by the strong dynamics close to the origin, $\langle X\rangle \ll M_{\mathrm{Pl}}$. $H_{J}$ is the Hubble rate in the Jordan frame, while $m_{1 \ell}^{J} \simeq 0.02 \lambda^{2} \Lambda$ denotes the effective Polonyi mass in the IYIT model. This mass is generated via meson loops at one-loop level [36]. The Polonyi field is stabilized at $\langle X\rangle$ as given in Eq. (8) only as long as $\langle X\rangle$ does not induce masses for the IYIT quarks larger than $\Lambda_{0}$. This defines a critical field value, $X_{c} \simeq \sqrt{2} / \lambda \Lambda_{0}$, above which the Polonyi potential changes from a quadratic to a logarithmic form. The requirement that $\langle X\rangle \lesssim X_{c}$ then translates into a lower bound, $\lambda_{\min }(\Lambda,\langle\rho\rangle) \sim 0.1 \cdots 1$, on $\lambda$. At the same time, we impose an upper bound, $\lambda \lesssim \lambda_{\text {pert }} \simeq 4$, so that noncalculable higher-dimensional terms in the Kähler potential, which scale like $\lambda^{2} /\left(16 \pi^{2}\right)$ [36], are suppressed by at least half an order of magnitude. In fact, for definiteness, we will set $\lambda^{2}=\lambda_{\min } \lambda_{\text {pert }}$ in the following. Meanwhile, a given value of $\lambda$ implies a lower bound on $\rho$, such that neither of $\lambda_{ \pm}$becomes larger than $\lambda_{\text {pert }}$. For our choice of $\lambda$, we find $\rho^{-2}<\rho_{\text {pert }}^{-2}=\left(\lambda_{\min } / \lambda_{\text {pert }}+\lambda_{\text {pert }} / \lambda_{\min }\right) / 2$, where $\rho_{\text {pert }}$ depends on the dynamical scale $\Lambda$ via $\lambda_{\text {min }}$.

The Polonyi field is a linear combination of the charge eigenstates $Z_{ \pm}$. It hence enters into the $\mathrm{D}$-term potential, where it mixes with the field $Y=\left(Z_{+}-Z_{-}\right) / \sqrt{2}$. This mixing destabilizes the vacuum in Eq. (8), unless $\left|\Delta m_{X Y}\right|^{2}=\left|g^{2} q_{0}^{2} \xi\right|<m_{1 \ell}^{J} m_{F}$, which translates into an upper bound on $g$. In our analysis of the inflationary dynamics, we will evaluate the bounds on $\rho$ and $g$ numerically. The lesson from these two bounds is the following: $\rho>\rho_{\text {pert }}$ guarantees that we can neglect nonperturbative corrections to the Kähler potential, while $g<g_{\max }$ ensures a stable vacuum in the SUSY-breaking sector.

The constant $w$ in Eq. (5) needs to be tuned to

$$
w_{0}=\left(1-\frac{4}{3 k}\right)^{1 / 2} \frac{\mu^{2} M_{\mathrm{Pl}}}{\sqrt{3}},
$$

so as to reach zero cosmological constant in the SUSYbreaking vacuum. We then obtain for $m_{3 / 2}=\langle W\rangle / M_{\mathrm{Pl}}^{2}$,

$$
m_{3 / 2}=\left(1-\frac{4}{3 k}\right)^{1 / 2} \frac{(1-f) k+2 / 3}{(1-f) k-4 / 3} \frac{\mu^{2}}{\sqrt{3} M_{\mathrm{Pl}}} .
$$

Making use of our choices for the parameters $\lambda$ and $\rho$, we arrive at the following phenomenological relation,

$$
\frac{m_{3 / 2}}{10^{11} \mathrm{GeV}} \sim\left(\frac{\sqrt{\xi}}{10^{15} \mathrm{GeV}}\right)^{5 / 2} \sim\left(\frac{\Lambda}{10^{15} \mathrm{GeV}}\right)^{5 / 2},
$$

which illustrates that SUSY is broken at a high scale. All mass scales in the IYIT sector are now solely controlled by $\Lambda$. This scale is dynamically generated via dimensional transmutation, meaning that our model does not require any hard dimensionful input scale. Eq. (12) sets the stage for our particular implementation of DHI.

We consider the following contributions to the kinetic function and superpotential from the inflationary sector,

$$
\begin{aligned}
F_{\mathrm{inf}} & =\frac{\chi}{2}\left(S^{\dagger}+S\right)^{2}-\frac{1}{2}(1-\chi)\left(S^{\dagger}-S\right)^{2}+\Phi^{\dagger} \Phi+\bar{\Phi}^{\dagger} \bar{\Phi}, \\
W_{\mathrm{inf}} & =\kappa S \Phi \bar{\Phi} .
\end{aligned}
$$

Taking $|\chi| \ll 1$, the real scalar component $\sigma$ of $S$ acquires an approximate shift symmetry and will play the role of the inflaton. The so-called waterfall fields $\Phi, \bar{\Phi}$ carry $B-L$ charges $\pm q$. Due to their $\sigma$-dependent mass spectrum, one of the scalar waterfall degrees of freedom becomes tachyonic at $\sigma \leq \sigma_{c}$, leading to a phase transition that ends inflation. The sequestered structure of Eq. (7) protects the waterfall fields from acquiring soft SUGRA masses of the order of $m_{3 / 2}$, which could prevent this phase transition. Note that since $B-L$ is already broken by $\left\langle M_{ \pm}\right\rangle \neq 0$ during inflation, the production of cosmic strings at the end of inflation can be avoided [19].

The scalar inflaton potential at $\sigma \gg \sigma_{c}$ is given by

$$
V(\sigma) \simeq \mathcal{C}^{4}(\sigma)\left[V_{D}^{J}+V_{F}^{J}(\sigma)+\frac{Q_{J}^{4}(\sigma)}{16 \pi^{2}} \ln (x(\sigma))\right],
$$


with the tree-level D- and F-term contributions being

$$
V_{D}^{J}=\frac{1}{2} q_{0}^{2} g^{2} \xi^{2}, \quad V_{F}^{J}(\sigma)=\frac{-f(\sigma)}{1-f(\sigma)} \mu^{4} .
$$

Similarly as in global SUSY, inflation is driven by the constant D-term potential induced by the FI term (4). $V_{F}^{J}$ arises due to F-term SUSY breaking in the hidden sector and vanishes in the true vacuum at $\sigma=0$. The function $f=(1-2 \chi) \chi \sigma^{2} /\left(3 M_{\mathrm{Pl}}^{2}\right) \ll 1$ is introduced below Eq. (8). The third term in Eq. (14) is the effective one-loop potential arising from integrating out the waterfall multiplets. Here, the renormalization scale $Q_{J}^{4}=q^{2} m_{D}^{4}+\delta m^{4}$ and $x=\left(m_{\text {eff }}^{2}+2 H_{J}^{2}\right) / Q_{J}^{2}$ are given in terms of the various contributions to the masses of the waterfall fields: $m_{D}^{2}=g^{2} q_{0} \xi$ is induced by the D term, $m_{\text {eff }}^{2}=\kappa^{2} \sigma^{2} / 2$ follows from the coupling in $W_{\mathrm{inf}}$, and $\delta m^{2} \simeq m_{3 / 2} m_{\text {eff }}$ is a bilinear soft mass in consequence of $R$ symmetry breaking. The critical inflaton field value $\sigma_{c}$ is obtained once $m_{\text {eff }}^{2}+2 H_{J}^{2}-Q_{J}^{2}=0$, which results in $\sigma_{c}^{2}=2 / \kappa^{2}\left(Q_{J}^{2}-2 H_{J}^{2}\right)$. The conformal factor $\mathcal{C}^{2}=-3 M_{\mathrm{Pl}}^{2} / \Omega_{\text {tot }}$ translates the Jordan-frame potentials to their counterparts in the Einstein frame.

Integrating out the heavy $B-L$ gauge fields results in additional gauge-mediated soft masses, $m_{\mathrm{gm}}$, for the waterfall fields [37] (see also [18]). However, at the level of the effective one-loop potential for the inflaton, these radiative corrections merely represent a two-loop effect. The inflaton itself, being a gauge singlet, receives by contrast no gauge-mediated soft mass. Moreover, one can show that, in the parameter range of interest, $m_{\mathrm{gm}}$ is always outweighed by the tree-level mass induced by the D-term potential, $m_{\mathrm{gm}} \lesssim 0.03 m_{D}$. For these reasons, we will neglect the effect of gauge mediation in the following.

We solve the slow-roll equation of motion numerically,

$$
K_{S^{\dagger} S}(\sigma) V(\sigma) \sigma^{\prime}(N)=M_{\mathrm{Pl}}^{2} V^{\prime}(\sigma),
$$

to obtain the predictions for the $\mathrm{CMB}$ observables at $N_{*} \simeq 55$ e-folds before the end of inflation. With

$$
\varepsilon=\frac{M_{\mathrm{Pl}}^{2}}{2}\left(\frac{V^{\prime}(\hat{\sigma})}{V}\right)^{2}, \quad \eta=M_{\mathrm{Pl}}^{2} \frac{V^{\prime \prime}(\hat{\sigma})}{V},
$$

where derivatives with respect to the canonically normalized field $\hat{\sigma}$ can be obtained by $\partial \hat{\sigma} / \partial \sigma=\sqrt{K_{S^{\dagger} S}}$, the amplitude of the scalar perturbation spectrum, its tilt and the tensor-to-scalar ratio are obtained as

$$
A_{s}=\frac{V}{24 \pi^{2} \varepsilon M_{P}^{4}}, \quad n_{s}=1-6 \varepsilon+2 \eta, \quad r=16 \varepsilon,
$$

evaluated at $\sigma\left(N_{*}\right)$. Requiring $A_{s}=A_{s}^{\text {obs }}=2.1 \times 10^{-9}[7]$ fixes $\Lambda$ (or equivalently $\xi$ ). The parameter $\kappa \neq 0$ explicitly breaks the shift symmetry in the superpotential, which leads us to expect that $\kappa \lesssim 1$. On the other hand, for $\kappa \ll 1$, the correct spectral index can only be obtained if the SUGRA contributions become much larger than the

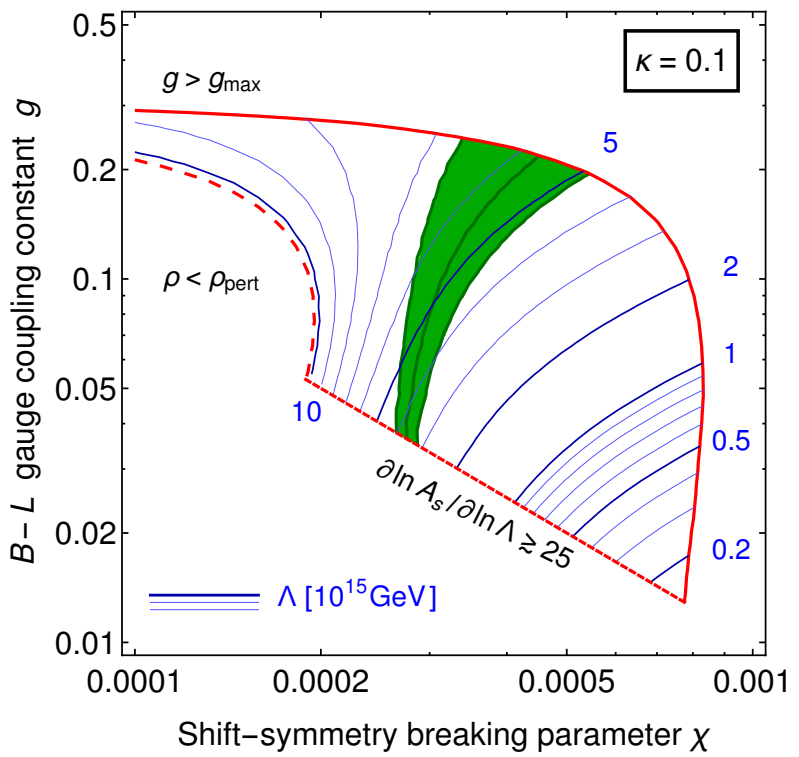

FIG. 1: Theoretical and experimental constraints. The green band indicates values of the spectral index in agreement (at $2 \sigma$ ) with the Planck 2015 data, $n_{s}=0.9677 \pm 0.006$ [7]. The blue contours indicate the values of the dynamical scale $\Lambda$ in the IYIT sector, such that $A_{s}=A_{s}^{\text {obs }}$. The red lines are boundaries of the parameter space due to the requirement of perturbativity $\left(\rho>\rho_{\text {pert }}\right)$ and a stable vacuum $\left(g<g_{\max }\right)$ in the SUSY-breaking sector as well as not too large fine-tuning in the parameters of the inflation sector $\left(\partial \ln A_{s} / \partial \ln \Lambda \lesssim 25\right)$.

one-loop contributions [27]. We thus set $\kappa=0.1$. In this regime, inflation occurs at field values slightly below the Planck scale. For simplicity, we also fix the $B-L$ charges to $q=2 q_{0}=-2$, inspired by neutrino mass generation (see below). We depict our results in the remaining $(\chi, g)$ plane in Fig. 1. $r$ is of $\mathcal{O}\left(10^{-6} \cdots 10^{-4}\right)$, which is, similarly as in FHI, far below current bounds.

These results are very well reproduced by approximate analytical expressions for the slow-roll parameters,

$$
\begin{aligned}
\varepsilon & \simeq\left(\frac{M_{\mathrm{Pl}}}{\sigma / \sqrt{2}}\right)^{2}\left[\left(1+\delta_{\varepsilon}^{4}\right) \frac{q^{2} g^{2}}{16 \pi^{2}} \frac{D_{0}^{2}}{V_{D}^{J}}-f \frac{F_{0}^{2}}{V_{D}^{J}}\right]^{2}, \\
-\eta & \simeq\left(\frac{M_{\mathrm{Pl}}}{\sigma / \sqrt{2}}\right)^{2}\left[\left(1-\delta_{\eta}^{4}\right) \frac{q^{2} g^{2}}{16 \pi^{2}} \frac{D_{0}^{2}}{V_{D}^{J}}+f \frac{F_{0}^{2}}{V_{D}^{J}}\right],
\end{aligned}
$$

with $F_{0}^{2}=\mu^{4} \gtrsim D_{0}^{2}=q_{0}^{2} g^{2} \xi^{2}$ and

$$
\left(\delta_{\varepsilon} / \delta\right)^{4}=\ln x+\frac{1}{2}, \quad\left(\delta_{\eta} / \delta\right)^{4}=\ln x+\frac{1}{2}+\frac{2+\delta^{4}}{1+\delta^{4}} .
$$

Here, $\delta^{2}=\delta m^{2} /\left(q m_{D}^{2}\right)$ parametrizes the effect of the soft B-term mass, $\delta m$, in the one-loop potential. Lowering the spectral index compared to DHI in global SUSY becomes possible due to the negative mass-squared induced by the SUGRA contributions to the tree-level F- 
term potential, reflected by the last term in Eq. (20),

$$
\Delta \eta \simeq-\frac{2 \chi}{3}\left(\frac{m_{3 / 2}}{H_{J}}\right)^{2}, \quad H_{J}^{2} \simeq \frac{V_{D}^{J}}{3 M_{\mathrm{Pl}}^{2}} .
$$

Successful inflation is thus due to the interplay of the oneloop contribution and the SUGRA-induced mass, with the latter being suppressed by an approximate shift symmetry, $\chi \sim 10^{-4}$. We note that $F_{\text {inf }} \supset \chi \sigma^{2}$ in Eq. (13) might, e.g., arise from further shift-symmetry breaking terms in the superpotential. Suppose the inflaton couples to superheavy multiplets with strength $\kappa^{\prime}$. Integrating out these fields results in an effective Kähler potential, $K_{1 \ell} \sim \kappa^{\prime 2} /\left(16 \pi^{2}\right)|S|^{2}$ [38]. With $\kappa^{\prime} \sim \kappa \sim 0.1$, this is of just the right order to explain the required value of $\chi$.

In the viable region of parameter space, inflation occurs either near a hill-top (i.e., a local maximum in the scalar potential) or near an inflection point, depending on the exact values of $\chi$ and $g$ [27]. The hill-top regime may suffer from an initial conditions problem. For particular parameter values, there is however a false vacuum at large field values. From there, $\sigma$ could tunnel to the correct side of the hill-top, thereby setting off inflation in our Universe. The inflection-point regime allows, by contrast, to start out at super-Planckian field values.

In FHI, $n_{s} \simeq 0.96$ is obtained from the interplay of the one-loop potential and the SUGRA tadpole, which is linear in the inflaton field. The tadpole also renders the question of initial conditions more subtle [10]. Its size is controlled by $m_{3 / 2}$, an independent parameter, which can be chosen in accord with low-scale SUSY breaking.

Let us conclude. We have presented a complete and phenomenologically viable SUGRA model of DHI, in which inflation is driven by the $\mathrm{D}$ term of a gauged $U(1)_{B-L}$ symmetry. Our model unifies the dynamics of dynamical SUSY breaking in the hidden sector, DHI, and spontaneous $B-L$ breaking. It links all relevant energy scales to the dynamical scale in the hidden sector, the magnitude of which is fixed by the amplitude of the CMB power spectrum, $\Lambda \simeq 5 \times 10^{15} \mathrm{GeV}$. This value is remarkably close to the GUT scale, $\Lambda_{\mathrm{GUT}} \sim 10^{16} \mathrm{GeV}$.

We based our construction on three assumptions: dynamical SUSY breaking as the origin of the FI term, Jordan-frame supergravity with canonical kinetic terms, and an approximate inflaton shift symmetry. These assumptions remedy all shortcomings of standard DHI: Our FI term is a field-dependent FI term. The associated modulus is stabilized via F-term SUSY breaking. The MSSM sfermions are stabilized against tachyonic D-terminduced masses thanks to their direct coupling to the hidden sector in the kinetic function $F_{\text {tot }}(7)$. Owing to our Jordan-frame description, the inflaton sector sequesters from the hidden sector, such that the fields in the inflation sector pick up no dangerous gravity-mediated soft masses. Meanwhile, a slight breaking of the shift symmetry provides a small SUGRA correction to the inflaton potential, $V \supset-\chi m_{3 / 2}^{2} \sigma^{2}$, that allows to reproduce $n_{s} \simeq 0.96$. As $B-L$ is spontaneously broken in the hidden sector already during inflation, no dangerous cosmic strings are produced during the waterfall transition.

Our model has important phenomenological consequences. For instance, if we assign $B-L$ charge $q=-2$ to the waterfall field $\Phi$, it can couple to the right-handed neutrinos $N_{i}$ in the seesaw extension of the MSSM, $W \supset h_{i j} / 2 \Phi N_{i} N_{j}$. For $q_{0} \xi<0$, it is the field $\Phi$ that acquires a nonzero VEV during the waterfall transition, $\langle\Phi\rangle=\left|q_{0} / q \xi\right|^{1 / 2}$, whereas $\langle\bar{\Phi}\rangle$ remains zero. This VEV generates the Majorana mass matrix for the righthanded neutrinos, $M_{i j}=h_{i j}\langle\Phi\rangle$, and, hence, sets the stage for the seesaw mechanism and leptogenesis [4]. Besides, our model predicts a superheavy SUSY mass spectrum. Only the lightest neutralino may have a fine-tuned small mass, so as to evade overproduction in gravitino decays [39]. Together with the corresponding chargino, this neutralino is then expected to be the only superparticle at low energies. It constitutes thermal neutralino dark matter and can be searched for in direct detection experiments. ${ }^{1}$ One may also hope to probe our model in gravitational-wave (GW) experiments. Depending on further model assumptions, the $B-L$ phase transition may give rise to observable signals [43]. Likewise, if the shift symmetry is realized along the imaginary component of $S$, the inflaton may have an axion-like coupling to gauge fields, $\mathcal{L}_{\text {eff }} \supset \operatorname{Im}(S) F \tilde{F}$. This could drastically enhance the GW signal from inflation [44].

Our model leaves open several questions that call for further exploration: For instance, one may ask what UV physics underlies the kinetic function in Eq. (7). It would be interesting to derive this structure from the viewpoint of a higher-dimensional brane-world scenario or from a strongly coupled conformal field theory [35]. We have only briefly sketched the mechanism of sfermion mass generation. It would, therefore, be desirable to devise a model that accounts for the origin of the scale $M_{*}$ in Eq. (7). These questions are however beyond the scope of this work. We conclude by stressing that our dynamical SUGRA model resolves all issues of standard DHI.

\footnotetext{
${ }^{1}$ In our model, the mediation of spontaneous SUSY breaking to the visible sector is essentially described by the framework of pure gravity mediation (PGM) [40]. In this mediation scheme, one is, e.g., able to achieve a light wino mass by tuning higgsino threshold corrections against the usual gaugino masses from anomaly mediation [41]. For $\tan \beta=v_{u} / v_{d} \simeq 1$ and Higgs mass parameters of the order of the gravity mass, $\left|\mu_{H}\right| \simeq|B| \simeq m_{3 / 2}$, PGM readily allows to push the wino mass down to $m_{\text {wino }} \simeq 2.7 \mathrm{TeV}$, so that it may constitute ordinary thermal dark matter in the form of weakly interacting massive particles (WIMPs). This finetuning might be the result of anthropic selection. In addition, the wino mass may also receive threshold and anomaly-mediated corrections from heavy vector matter multiplets charged under $S U(2)_{L}$ and/or threshold corrections from the $\mathrm{F}$ terms of flat directions in KSVZ-type axion models [42]. In this case, these contributions would also play a role in tuning the wino mass.
} 
The authors wish to thank T. T. Yanagida for inspiring discussions at Kavli IPMU at the University of Tokyo in the early stages of this project in 2014. The authors are grateful to B. v. Harling and L. Witkowski for helpful remarks and thank W. Buchmüller, E. Kiritsis and T. T. Yanagida for useful comments on the manuscript. V.D. acknowledges financial support from the UnivEarthS Labex program at Sorbonne Paris Cité (ANR-10-LABX-0023 and ANR-11-IDEX-0005-02) and the Paris Centre for Cosmological Physics. This project has received support from the European Union's Horizon 2020 research and innovation programme under the Marie Skłodowska-Curie grant agreement No. 674896 (K. S.). K. S. acknowledges the hospitality of the APC/PCCP group during a stay at Paris Diderot University, where this project was finished.

[1] A. H. Guth, The Inflationary Universe: A Possible Solution to the Horizon and Flatness Problems, Phys. Rev. D 23, 347 (1981); A. D. Linde, A New Inflationary Universe Scenario: A Possible Solution of the Horizon, Flatness, Homogeneity, Isotropy and Primordial Monopole Problems, Phys. Lett. B 108, 389 (1982); A. Albrecht and P. J. Steinhardt, Cosmology for Grand Unified Theories with Radiatively Induced Symmetry Breaking, Phys. Rev. Lett. 48, 1220 (1982).

[2] D. H. Lyth and A. Riotto, Particle physics models of inflation and the cosmological density perturbation, Phys. Rept. 314, 1 (1999), HEP-PH/9807278; A. D. Linde, Particle physics and inflationary cosmology, Contemp. Concepts Phys. 5, 1 (1990), HEP-TH/0503203.

[3] A. D. Linde, Axions in inflationary cosmology, Phys. Lett. B 259, 38 (1991); Hybrid inflation, Phys. Rev. D 49, 748 (1994), ASTRO-PH/9307002.

[4] W. Buchmuller, K. Schmitz and G. Vertongen, Matter and Dark Matter from False Vacuum Decay, Phys. Lett. B 693, 421 (2010), ARXIV:1008.2355 [HEP-PH]; Entropy, Baryon Asymmetry and Dark Matter from Heavy Neutrino Decays, Nucl. Phys. B 851, 481 (2011), ARXIV:1104.2750 [HEP-PH]; W. Buchmuller, V. Domcke and K. Schmitz, Spontaneous B-L Breaking as the Origin of the Hot Early Universe, Nucl. Phys. B 862, 587 (2012), ARXIV:1202.6679 [HEP-PH]; WIMP Dark Matter from Gravitino Decays and Leptogenesis, Phys. Lett. B 713, 63 (2012), ARXIV:1203.0285 [HEP-PH]; W. Buchmuller, V. Domcke, K. Kamada and K. Schmitz, The Gravitational Wave Spectrum from Cosmological B-L Breaking, JCAP 1310, 003 (2013), ARXIV:1305.3392 [HEP-PH]; A Minimal Supersymmetric Model of Particle Physics and the Early Universe, arXiv:1309.7788 [hepph]; K. Schmitz, The B-L Phase Transition: Implications for Cosmology and Neutrinos, arXiv:1307.3887 [hep-ph]; V. Domcke, Matter, Dark Matter and Gravitational Waves from a GUT-Scale U(1) Phase Transition, DESY-THESIS-2013-037.

[5] P. Minkowski, $\mu \rightarrow$ er at a Rate of One Out of $10^{9}$ Muon Decays?, Phys. Lett. 67B, 421 (1977); T. Yanagida, Horizontal Symmetry And Masses Of Neutrinos, Conf.
Proc. C 7902131, 95 (1979); Horizontal Symmetry and Masses of Neutrinos, Prog. Theor. Phys. 64, 1103 (1980); M. Gell-Mann, P. Ramond and R. Slansky, Complex Spinors and Unified Theories, Conf. Proc. C 790927, 315 (1979), ARXIV:1306.4669 [HEP-TH]; R. N. Mohapatra and G. Senjanovic, Neutrino Mass and Spontaneous Parity Violation, Phys. Rev. Lett. 44, 912 (1980).

[6] M. Fukugita and T. Yanagida, Baryogenesis Without Grand Unification, Phys. Lett. B 174, 45 (1986).

[7] P. A. R. Ade et al., [PLANCK Collaboration], Planck 2015 results. XX. Constraints on inflation, Astron. Astrophys. 594, A20 (2016), ARXIV:1502.02114 [ASTROPH.CO].

[8] E. J. Copeland, A. R. Liddle, D. H. Lyth, E. D. Stewart and D. Wands, False vacuum inflation with Einstein gravity, Phys. Rev. D 49, 6410 (1994), ASTroPH/9401011; G. R. Dvali, Q. Shafi and R. K. Schaefer, Large scale structure and supersymmetric inflation without fine tuning, Phys. Rev. Lett. 73, 1886 (1994), HEP-PH/9406319.

[9] P. Binetruy and G. R. Dvali, D term inflation, Phys. Lett. B 388, 241 (1996), HEP-PH/9606342; E. Halyo, Hybrid inflation from supergravity D terms, Phys. Lett. B 387, 43 (1996), HEP-PH/9606423.

[10] W. Buchmuller, L. Covi and D. Delepine, Inflation and supersymmetry breaking, Phys. Lett. B 491, 183 (2000), HEP-PH/0006168; K. Nakayama, F. Takahashi and T. T. Yanagida, Constraint on the gravitino mass in hybrid inflation, JCAP 1012, 010 (2010), ARXIV:1007.5152 [HEP-PH]; W. Buchmuller, V. Domcke, K. Kamada and K. Schmitz, Hybrid Inflation in the Complex Plane, JCAP 1407, 054 (2014), ARXIV:1404.1832 [HEP-PH].

[11] P. Fayet and J. Iliopoulos, Spontaneously Broken Supergauge Symmetries and Goldstone Spinors, Phys. Lett. 51B, 461 (1974).

[12] Z. Komargodski and N. Seiberg, Comments on the FayetIliopoulos Term in Field Theory and Supergravity, JHEP 0906, 007 (2009), ARXIV:0904.1159 [HEP-TH]; K. R. Dienes and B. Thomas, On the Inconsistency of FayetIliopoulos Terms in Supergravity Theories, Phys. Rev. D 81, 065023 (2010), ARXIV:0911.0677 [HEP-TH].

[13] T. Banks and N. Seiberg, Symmetries and Strings in Field Theory and Gravity, Phys. Rev. D 83, 084019 (2011), ARXIV:1011.5120 [HEP-TH].

[14] M. Dine, N. Seiberg and E. Witten, Fayet-Iliopoulos Terms in String Theory, Nucl. Phys. B 289, 589 (1987).

[15] Z. Komargodski and N. Seiberg, Comments on Supercurrent Multiplets, Supersymmetric Field Theories and Supergravity, JHEP 1007, 017 (2010), ARXIV:1002.2228 [HEP-TH].

[16] G. D. Coughlan, W. Fischler, E. W. Kolb, S. Raby and G. G. Ross, Cosmological Problems for the Polonyi Potential, Phys. Lett. 131B, 59 (1983); J. R. Ellis, D. V. Nanopoulos and M. Quiros, On the Axion, Dilaton, Polonyi, Gravitino and Shadow Matter Problems in Supergravity and Superstring Models, Phys. Lett. B 174, 176 (1986).

[17] P. Binetruy, G. Dvali, R. Kallosh and A. Van Proeyen, Fayet-Iliopoulos terms in supergravity and cosmology, Class. Quant. Grav. 21, 3137 (2004), HEP-TH/0402046; C. Wieck and M. W. Winkler, Inflation with FayetIliopoulos Terms, Phys. Rev. D 90, 103507 (2014), ARXIV:1408.2826 [HEP-TH]. 
[18] K. S. Babu, K. Schmitz and T. T. Yanagida, Pure gravity mediation and spontaneous $B-L$ breaking from strong dynamics, Nucl. Phys. B 905, 73 (2016), ARXIV:1507.04467 [HEP-PH].

[19] V. Domcke, K. Schmitz and T. T. Yanagida, Dynamical D-Terms in Supergravity, Nucl. Phys. B 891, 230 (2015), ARXIV:1410.4641 [HEP-TH].

[20] T. T. Dumitrescu, Z. Komargodski and M. Sudano, Global Symmetries and D-Terms in Supersymmetric Field Theories, JHEP 1011, 052 (2010), ARXIV:1007.5352 [HEP-TH]; Y. Kawamura, Limitation on Magnitude of D-components, Prog. Theor. Phys. 125, 509 (2011), ARXIV:1008.5223 [HEP-PH].

[21] H. P. Nilles, Supersymmetry, Supergravity and Particle Physics, Phys. Rept. 110, 1 (1984).

[22] M. Dine, W. Fischler and D. Nemeschansky, Solution of the Entropy Crisis of Supersymmetric Theories, Phys. Lett. 136B, 169 (1984); G. D. Coughlan, R. Holman, P. Ramond and G. G. Ross, Supersymmetry and the Entropy Crisis, Phys. Lett. 140B, 44 (1984).

[23] O. Seto and J. Yokoyama, Hiding cosmic strings in supergravity D-term inflation, Phys. Rev. D 73, 023508 (2006), HEP-PH/0508172; C. M. Lin and J. McDonald, Supergravity modification of D-term hybrid inflation: Solving the cosmic string and spectral index problems via a right-handed sneutrino, Phys. Rev. D 74, 063510 (2006), HEP-PH/0604245; J. Rocher and M. Sakellariadou, Dterm inflation in non-minimal supergravity, JCAP 0611 , 001 (2006), HEP-TH/0607226; W. Buchmuller, V. Domcke and K. Schmitz, Superconformal D-Term Inflation, JCAP 1304, 019 (2013), ARXIV:1210.4105 [HEP$\mathrm{PH}]$. W. Buchmuller, V. Domcke and K. Kamada, The Starobinsky Model from Superconformal D-Term Inflation, Phys. Lett. B 726, 467 (2013), ARXIV:1306.3471 [HEP-TH].

[24] P. A. R. Ade et al. [PLANCK Collaboration], Planck 2013 results. XXV. Searches for cosmic strings and other topological defects, Astron. Astrophys. 571, A25 (2014), ARXIV:1303.5085 [ASTRO-PH.CO].

[25] S. Ferrara, R. Kallosh, A. Linde, A. Marrani and A. Van Proeyen, Jordan Frame Supergravity and Inflation in NMSSM, Phys. Rev. D 82, 045003 (2010), ARXIV:1004.0712 [HEP-TH]; Superconformal Symmetry, NMSSM, and Inflation, Phys. Rev. D 83, 025008 (2011), ARXIV:1008.2942 [HEP-TH]; S. Ferrara, R. Kallosh, A. Linde and M. Porrati, Minimal Supergravity Models of Inflation, Phys. Rev. D 88, 085038 (2013), ARXIV:1307.7696 [HEP-TH].

[26] M. Kawasaki, M. Yamaguchi and T. Yanagida, Natural chaotic inflation in supergravity, Phys. Rev. Lett. 85, 3572 (2000), HEP-PH/0004243.

[27] V. Domcke and K. Schmitz, Inflation from High-Scale Supersymmetry Breaking, Phys. Rev. D 97, 115025 (2018), ARXIV:1712.08121 [HEP-PH].

[28] K. I. Izawa and T. Yanagida, Dynamical supersymmetry breaking in vector-like gauge theories, Prog. Theor. Phys. 95, 829 (1996), HEP-TH/9602180; K. A. Intriligator and S. D. Thomas, Dynamical supersymmetry breaking on quantum moduli spaces, Nucl. Phys. B 473, 121 (1996), HEP-TH/9603158.

[29] A. Manohar and H. Georgi, Chiral Quarks and the Nonrelativistic Quark Model, Nucl. Phys. B 234, 189 (1984); H. Georgi and L. Randall, Flavor Conserving CP Violation in Invisible Axion Models, Nucl. Phys. B 276, 241
(1986); A. G. Cohen, D. B. Kaplan and A. E. Nelson, Counting 4 pis in strongly coupled supersymmetry, Phys. Lett. B 412, 301 (1997), HEP-PH/9706275; M. A. Luty, Naive dimensional analysis and supersymmetry, Phys. Rev. D 57, 1531 (1998), HEP-PH/9706235.

[30] N. Seiberg, Exact results on the space of vacua of fourdimensional SUSY gauge theories, Phys. Rev. D 49, 6857 (1994), HEP-TH/9402044.

[31] L. O'Raifeartaigh, Spontaneous Symmetry Breaking for Chiral Scalar Superfields, Nucl. Phys. B 96, 331 (1975).

[32] K. Harigaya, M. Ibe, K. Schmitz and T. T. Yanagida, Peccei-Quinn Symmetry from Dynamical Supersymmetry Breaking, Phys. Rev. D 92, 075003 (2015), ARXIV:1505.07388 [HEP-PH].

[33] K. Schmitz and T. T. Yanagida, Dynamical supersymmetry breaking and late-time $R$ symmetry breaking as the origin of cosmic inflation, Phys. Rev. D 94, 074021 (2016), ARXIV:1604.04911 [HEP-PH].

[34] J. Polonyi, Generalization of the Massive Scalar Multiplet Coupling to the Supergravity, Hungary Central Inst. Res. (1978), KFKI-1977-93 (unpublished).

[35] K. Inoue, M. Kawasaki, M. Yamaguchi and T. Yanagida, Vanishing squark and slepton masses in a class of supergravity models, Phys. Rev. D 45, 328 (1992); L. Randall and R. Sundrum, Out of this world supersymmetry breaking, Nucl. Phys. B 557, 79 (1999), HEPтн/9810155; M. A. Luty and R. Sundrum, Supersymmetry breaking and composite extra dimensions, Phys. Rev. D 65, 066004 (2002), HEP-TH/0105137; Anomaly mediated supersymmetry breaking in four-dimensions, naturally, Phys. Rev. D 67, 045007 (2003), HEP-TH/0111231; M. Ibe, K.-I. Izawa, Y. Nakayama, Y. Shinbara and T. Yanagida, Conformally sequestered SUSY breaking in vector-like gauge theories, Phys. Rev. D 73, 015004 (2006), HEP-PH/0506023; More on conformally sequestered SUSY breaking, Phys. Rev. D 73, 035012 (2006), HEP-PH/0509229.

[36] Z. Chacko, M. A. Luty and E. Ponton, Calculable dynamical supersymmetry breaking on deformed moduli spaces, JHEP 9812, 016 (1998), HEP-TH/9810253.

[37] K. Intriligator and M. Sudano, General Gauge Mediation with Gauge Messengers, JHEP 1006, 047 (2010), ARXIV:1001.5443 [HEP-PH].

[38] M. K. Gaillard and V. Jain, Supergravity coupled to chiral matter at one loop, Phys. Rev. D 49, 1951 (1994), HEP-TH/9308090; E. D. Stewart, Flattening the inflaton's potential with quantum corrections, Phys. Lett. B 391, 34 (1997), HEP-PH/9606241; Flattening the inflaton's potential with quantum corrections. 2., Phys. Rev. D 56, 2019 (1997), HEP-PH/9703232.

[39] T. Moroi and L. Randall, Wino cold dark matter from anomaly mediated SUSY breaking, Nucl. Phys. B 570, 455 (2000), HEP-PH/9906527; K. S. Jeong and F. Takahashi, A Gravitino-rich Universe, JHEP 1301, 173 (2013), ARXIV:1210.4077 [HEP-PH]; T. Moroi, M. Nagai and M. Takimoto, Non-Thermal Production of Wino Dark Matter via the Decay of Long-Lived Particles, JHEP 1307, 066 (2013), ARXIV:1303.0948 [HEP-PH].

[40] M. Ibe, T. Moroi and T. T. Yanagida, Possible Signals of Wino LSP at the Large Hadron Collider, Phys. Lett. B 644, 355 (2007), HEP-PH/0610277; M. Ibe and T. T. Yanagida, The Lightest Higgs Boson Mass in Pure Gravity Mediation Model, Phys. Lett. B 709, 374 (2012), ARXIV:1112.2462 [HEP-PH]; N. Arkani-Hamed, 
A. Gupta, D. E. Kaplan, N. Weiner and T. Zorawski, Simply Unnatural Supersymmetry, ARXIV:1212.6971 [HEP-PH].

[41] M. Ibe, S. Matsumoto and T. T. Yanagida, Pure Gravity Mediation with $m_{3 / 2}=10-100$ TeV, Phys. Rev. D 85, 095011 (2012), ARXIV:1202.2253 [HEP-PH].

[42] K. Harigaya, M. Ibe and T. T. Yanagida, A Closer Look at Gaugino Masses in Pure Gravity Mediation Model / Minimal Split SUSY Model, JHEP 1312, 016 (2013), ARXIV:1310.0643 [HEP-PH].

[43] M. Maggiore, Gravitational wave experiments and early universe cosmology, Phys. Rept. 331, 283 (2000), GRQC/9909001.

[44] J. L. Cook and L. Sorbo, Particle production during inflation and gravitational waves detectable by groundbased interferometers, Phys. Rev. D 85, 023534 (2012), Erratum ibid. D 86, 069901 (2012), ARXIV:1109.0022 [Astro-Ph.CO]; N. Barnaby, E. Pajer and M. Peloso, Gauge Field Production in Axion Inflation: Consequences for Monodromy, non-Gaussianity in the CMB, and Gravitational Waves at Interferometers, Phys. Rev. D 85, 023525 (2012), ARXIV:1110.3327 [ASTRO-PH.CO]; V. Domcke, M. Pieroni and P. Binetruy, Primordial gravitational waves for universality classes of pseudoscalar inflation, JCAP 1606, 031 (2016), ARXIV:1603.01287 [ASTRO-PH.CO]. 